\title{
Recent Modeling of MDMO-PPV: PCBM Solar Cells Versus Mixture Ratio, Electric Field and Incidence Angle with Hopping Model
}

\author{
M.R. Merad Boudia ${ }^{1}$, A.Cheknane $^{2}$, B.Benyoucef ${ }^{1}$, A.M Ferouani ${ }^{1}$ \\ and H. S. Hilal ${ }^{3}$ \\ ${ }^{1}$ University AbouBakerBelkaid of Tlemcen, URMER, Tlemcen, Algeria, \\ ${ }^{2}$ University Amar Telidji of Laghouat, LSDSDM, Laghouat, Algeria, \\ ${ }^{3}$ SSERL, Chemistry, An-Najah N. University, Nablus, Palestine
}

Corresponding Author:

Email: mustapha.merad@gmail.com

Keyword: Hopping model, organic polymer solar cells, electric field, mixture ratio

\begin{abstract}
It is a matter of controversy why excitons can efficiently dissociate into free carriers at an intrinsic polymer/fullerene interface. While extensive characterization is performed in the course of many reported experimental studies, correlation of performance and physical parameters among studies done in different laboratories is low, pointing out the need to address some aspects of BHJSC active materials that have received relatively some attention.

In this paper, we discuss the modeling of MDMO-PPV/PCBM(Poly(2-methoxy-5-(3,7dimethyloctyloxy)-1,4-phenylenevinylene)/ soluble C60 derivative, methanofullerene, [6,6]-phenyl C61-butyric acid methyl ester organic devices. In our approach, we apply two recent methods so called transfer matrix method and hopping model to calculate the exciton dissociation probability, and photocurrent density versus mixture ratio, electric field and angle of incidence. The results show that EDP (exciton dissociation probability) in solar cells without PEDOT-PSS (Poly (ethylendioxythiophene)-Poly(styrene sulfonic acid)) HTL hole transporting layer is better than the cells with additional layer in enhancing the performance of MDMO-PPV/PCBM solar cells. When the weight ratio of MDMO-PPV is less than 3:5 and $2: 5$ respectively, the best exciton dissociation probability, and photocurrent density of solar cell is obtained
\end{abstract}

\section{INTRODUCTION}

Polymer solar cells (PSCs), considered as a promising source of renewable energy, have attracted much attention because of their low-cost, compatibility with flexible substrates and large-area applications[1,4]. Recently, the power conversion efficiency of the bulk heterojunction (BHJ) PSCs around $7.4 \%$ has been realized $[5,6]$.

The photovoltaic effect involves the generation of electron and hole pairs and their subsequent collection at the opposite electrodes. In inorganic materials, the photon absorption produces free charge directly. The conjugated semiconducting polymers used in photovoltaic typically have low dielectric constants, which causes Frenkelexcitons to be created upon light absorption [7]. These Coulombically bound electron-hole pairs have a binding energy of approximately $0.5 \mathrm{eV}$ [8]. Higher efficiency devices were prepared from MDMO-PPV/[6]PCBM blends reaching 3.1\% in the so-called bulk heterojunction geometry, PEDOT: PSS (Figure.1) is solution processible and possesses a suitable work function to act as an effective hole transporting layer in the word of organic solar cells[9]. 

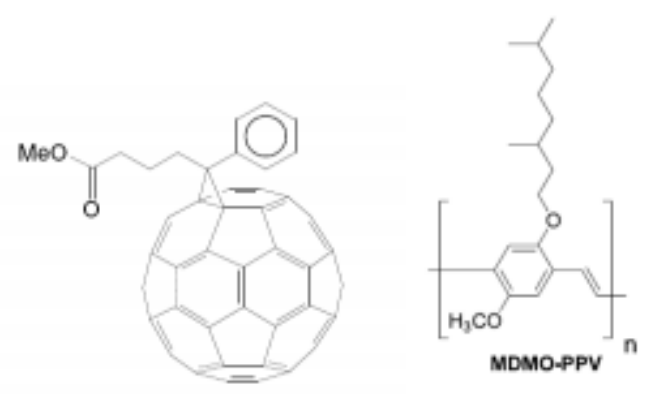

(a)

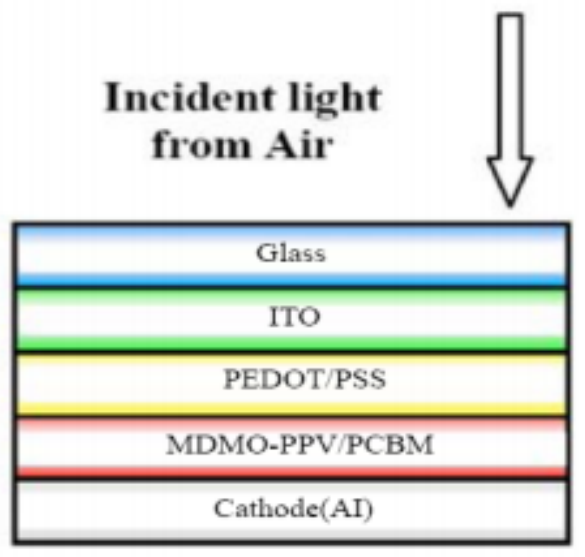

(b)

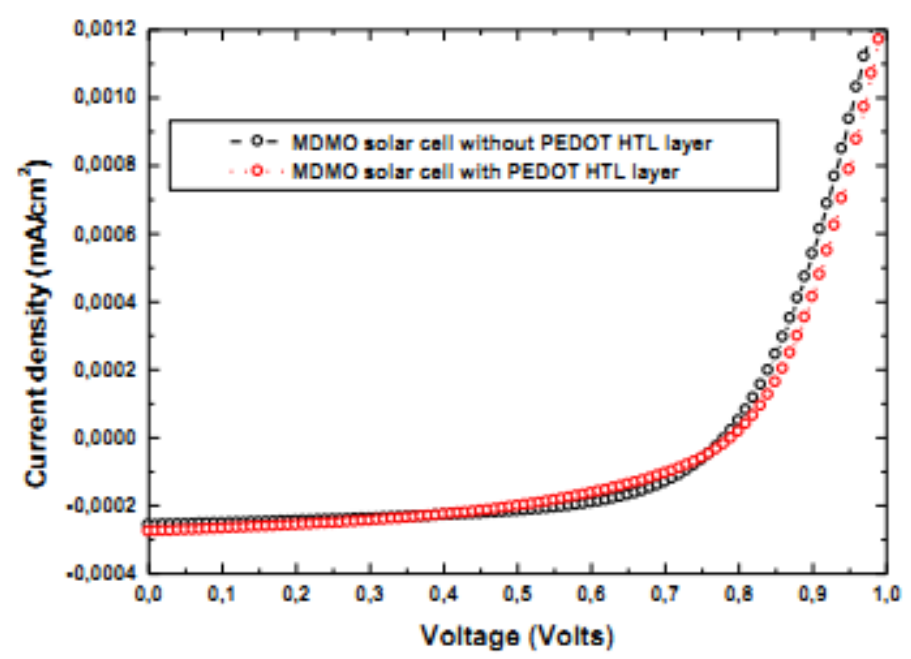

(c)

Figure 1 Chemical structures of the used conjugated organic polymers, namely, PCBM(C60) and MDMO-PPV (a) Schematical diagram of an organic solar cell (b) Current density versus bias of the studied solar cells (c ) [9]

In this paper we present an overview of recent achievements in experimental and theoretical studies of a hopping transport mechanism and there relaxation parameters by an analytical model, which is done in the next section introducing mixture ratio and incidence angle in addition to electric field contributed by S.D Baranovskii et al [13]. Finally, outlooks for the development of organic electronic devices based on the studied materials are given. 


\section{Model and calculation method}

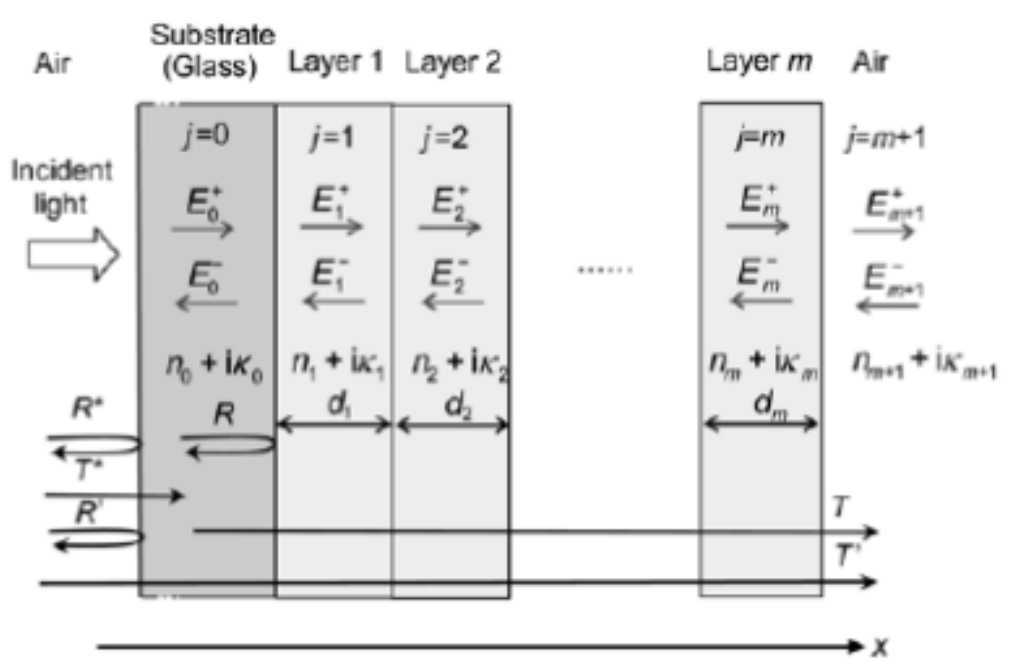

Figure 2 Geometry of a general multilayer structure [12]

To investigate the effect of applying an HTL layer to a cell as described above, a method of determining the optical transmission and reflection properties of a multilayer system is required that also includes the interference effects. The approach used here is the multiplication of the matrixes [9] that describe how the wave propagates by the whole device. Figure. 2 shows the arrangement of propagation of the forwards and towards fields in the back of the device under AM 1.5 solar spectrum.

We start by decomposing the optic electrical field into an upstream and a downstream component [10]. The equation (1) gives the sum of the electric field in the positive and the negative directions respectively as

$$
E_{j}(x)=E_{j}^{+}(x)+E_{j}^{-}(x)
$$

The total system transfer matrix Swich relates the electric field between the substrate $(\mathrm{j}=\mathrm{O})$ and ambient side $(\mathrm{j}=\mathrm{m}+1)$ by

$$
\left[\begin{array}{c}
E_{0}^{+} \\
E_{0}^{-}
\end{array}\right]=S\left[\begin{array}{l}
E_{m+1}^{+} \\
E_{m+1}^{-}
\end{array}\right]
$$

where $\mathrm{S}$ is the product of all interface and layer matrices that light propagates through orderly.

$$
\begin{aligned}
& {\left[\begin{array}{ll}
S_{11} & S_{12} \\
S_{21} & S_{22}
\end{array}\right]=S\left[\begin{array}{l}
E_{m+1}^{+} \\
E_{m+1}^{-}
\end{array}\right]} \\
& T^{\prime}=\frac{T^{*} T}{1+R^{*} R} \\
& R^{\prime}=\frac{R^{*}+R}{1+R^{*} R}
\end{aligned}
$$

with

$$
T^{*}=\left[2 /\left(1+n_{0}\right)\right]^{2}
$$

and

$$
R^{*}=\left[\left(1-n_{0}\right) /\left(1+n_{0}\right)\right]^{2}
$$


According to Fresnel relation for transmission and reflection and $\mathrm{n} 0$ is the real index of reflection of the substrate.

For other details, the readers can overview references [10-11].

The calculation of the exciton dissociation probability is according to the following equation [13]

$$
\eta(E) \approx\left[1+\left(v_{0} \tau_{0}\right)^{-1} \exp \left(\frac{-E_{b}-\beta \sqrt{E}}{k_{B} T}\right)\right]^{-1}
$$

$C=\frac{e^{2}}{4 \pi \varepsilon \varepsilon_{0} k_{B} T r_{0}}$,

$$
B=\frac{e E r_{0}}{k_{B} T},
$$

and $\quad \beta=\left(\frac{e^{3}}{\pi \varepsilon \varepsilon_{0}}\right)^{1 / 2}$

where

$v_{0}$ is the attempt-to-escape frequency, $E_{b}$ is the exciton binding energy,

$k_{B}$ is the Boltzmann constant,

$T$ is the temperature,

$e$ is the elementary charge,

$\varepsilon$ is the dielectric constant,

$\varepsilon_{0}$ is the permittivity of vacuum,

$r_{0}=1 \mathrm{~nm}$.

The mixture molar ratio of the donor to the totals implemented in the hopping model for the first time is defined as [14]

$$
\zeta=\frac{\varepsilon-\varepsilon_{H O S}}{\varepsilon_{D O P}-\varepsilon_{H O S}}
$$

where $\varepsilon_{D O P}$ and $\varepsilon_{H O S}$ are the dopant and host dielectric constant, respectively.

The photogenerated current is equal as given in [17]

$$
J_{p h}=e G L\left[\frac{\exp \left(e V / k_{B} T\right)+1}{\exp \left(e V / k_{B} T\right)-1}-\frac{2 k_{B} T}{e V}\right]
$$

where

$V$ is the effctivepotensial,

$k_{B}$ the Boltzman constant,

$T$ the temperature

$e G L$ represents the saturated photocurrent.

\section{Results and discussion}

MDMO-PPV composite materials were evaluated as electron donor/acceptor materials in ITO-free organic bulk heterojunction solar cells by using glass as substrates. We are interested in this work to the mixture ratio in order to optimize exciton dissociation probability (EDP) and photocurrent of MDMO-PPV based solar cells using analytical hopping model, where physical constants are taken from $[13,14,15]$ and wavelenght was fixed at $600 \mathrm{~nm}[16]$. 


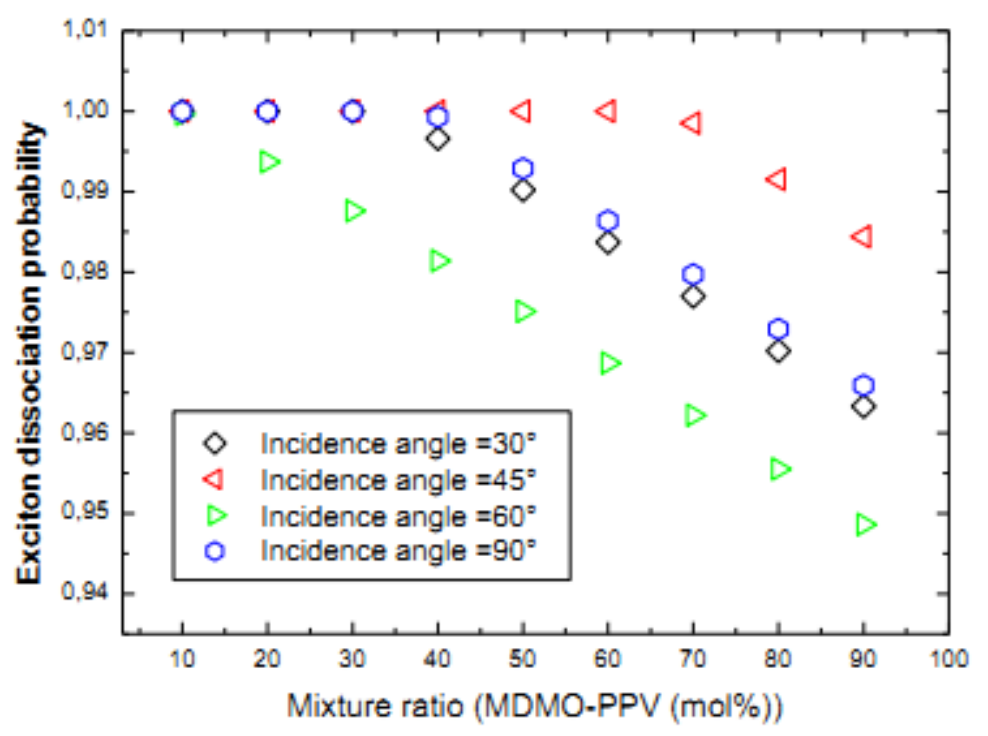

Figure 3 Exciton dissociation probabilty versus mixture ratio at different angles of incidence with PEDOT/PSS additional layer

Figure 3 show that the best values of the EDP are reported for less than $40 \%$ of mixture rato at angle of incidence $45^{\circ}, 90^{\circ}$ and $30^{\circ}$ respectively. Figure 4 shows that the best values of the EDP are reported for less than $60 \%$ of mixture ratio at angle of incidence $60^{\circ}$ and $90^{\circ}$ respectively when EDP is acceptable for $30^{\circ}$ and $45^{\circ}$. The clear difference is the effect of the PEDOT: PSS layer when it affect the dissociation probability earlier (decreasing rapidly at mixture ratio more than $45 \%$ ). Several electrical properties are reducing at this range in the work presented by[14].

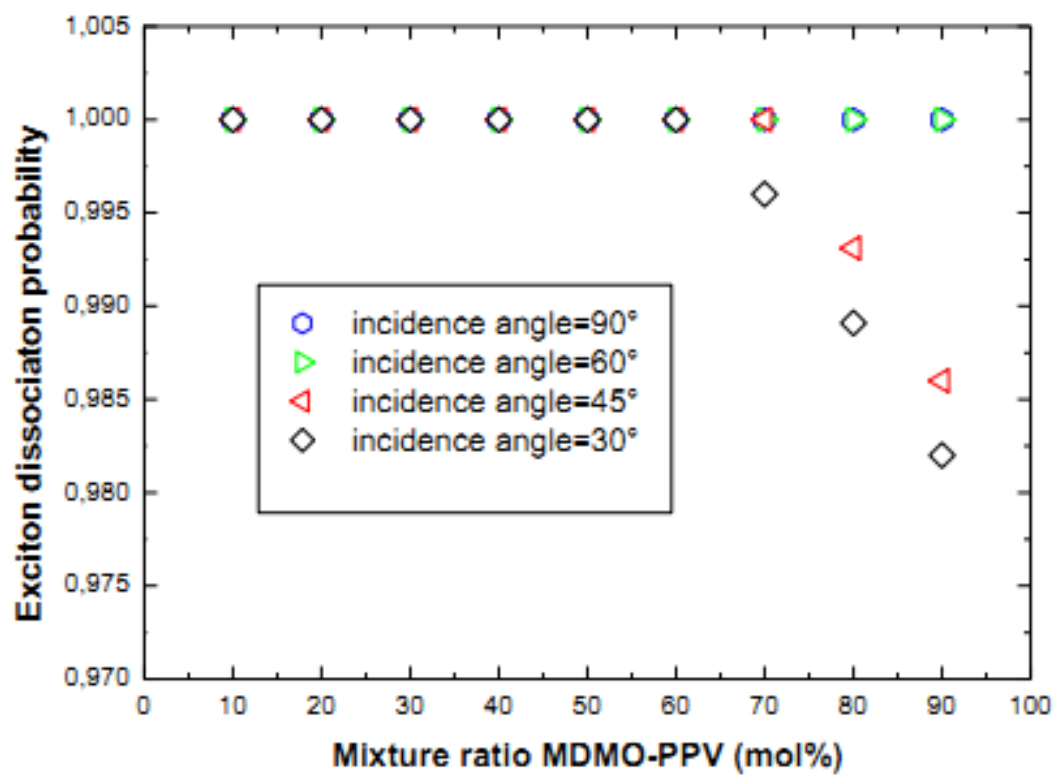

Figure 4 Exciton dissociation probability versus mixture ratio at different angles of incidence without PEDOT/PSS additional layer

From the results reported here and by ploting the photocurrent density versus electric field in a loglog diagram, we show that a good linear correlation between the last parameters was governed, and a general equation modeling the phenomena for all incidence angles is written as

$$
\frac{J_{p h 1}}{J_{p h 2}}=0.87
$$


$J_{p h 1}$ and $J_{p h 2}$ are the calculated photocurrent for solar cells with and whithout additional layer in PEDOT: PSS respectively.

The same result $(\sim 0.89)$ is giving by dividing experimental values of the two cells fabricated by [9] which the thickness of the HTL layer is $140 \mathrm{~nm}$ at $V=1$ volt. The majority of the photocurrent optimizations results (Figure.5) are given by 0.9 of the mixture ratio and $r_{0}=1.1 \mathrm{~nm}$ for any incidence angle.

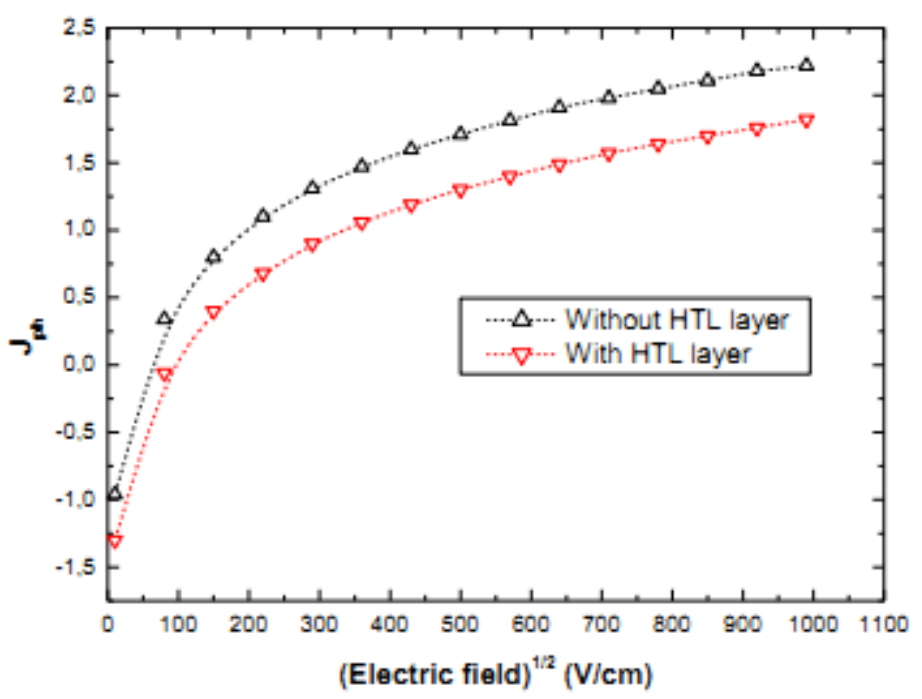

Figure 5 Normalized photocurrent versus electric field with the following parameters: Incidence angle is $30^{\circ} \lambda=600 \mathrm{~nm}$ and $\mathrm{V}=1$ volt with/without HTL layer.

\section{Conclusion}

In conclusion, we have shown that the electric field dependence of MDMO-PPV/PCBM based solar cells, in the range from 0 to $1000 \mathrm{~V} / \mathrm{cm}$, can be used in terms of a model coupling mixture ratio of donor materials and incidence angle by hopping transport to enhance exciton dissociation probability and photogenerated current density in disordered materials. We show that the best exciton dissociation probability is performed at less than $40 \%$ of mixture ratio for solar cells with and without HTL layer. The same analytical model that covers all relevant processes among photons, excitons and charges provides a powerful framework for extracting material and device parameters by a combined experimental and simulation approach.

\section{References}

[1] Yu G, Gao J, Hummelen J C, Wudl F and Heeger A J 1995 Science 2701789

[2] Reyes-Reyes M, Kim K and Carroll D L 2005 Appl. Phys. Lett. 87083506

[3] Kim J Y, Lee K, Coates N E, Moses D, Nguyen T Q, Dante M and Heeger A J 2007 Science 317222

[4] Dennler G, Scharber M C and Brabec C J 2009 Adv. Mater. 211323

[5] Chen H Y, Hou J H, Zhang S Q, Liang Y Y, Yang G W, Yang Y, Yu L P, Wu Y and Li G 2009 Nature Photon. 3649

[6] Zhao G, He Y and Li Y $20106.5 \%$ efficiency of polymère solar cells based on poly(3 hexylthiophene) and indene-C60 bisadduct by device optimization Adv. Mater. 22 4355-8

[7] Frenkel J 1931 On the transformation of light into heat in solids. I Phys. Rev. 37 17-44

[8] Arkhipov V I and Bassler H 2004 Exciton dissociation and charge photogeneration in pristine and doped conjugated polymers Phys. Status Solidi a 201 1152-87

[9] T. Aernouts, W. Geens, J. Poortmans, P. Heremans, S. Borghs and R. Mertens, 'Extraction of Bulk and Contact Components of the Series Resistances in Organic Bulk Donor-Acceptor Heterojunctions', Thin Solid Films Vol. 403/404, pp. 297 - 301, 2002. 
[10] L. A. A. Pettersson, S. Ghosh and O. Inganas, Org. Electron. 3 (2002), 143.

[11] Nils-KristerPersson, Hans Arwin, OlleInganas «Optical optimization of polyfluorene-fullerene blend photodiodes» JOURNAL OF APPLIED PHYSICS 97, 034503 (2005)

[12] Z.X Yan, MI BaoXio G ZhiQiang, H.Wei, «Recent progress in numerical modeling for organic thin film solar cells » science china review in Physics Mechanics and Astronomy vol 54 n)3 375 $387(2010)$

[13] O. Rubel, S. D. Baranovskii, W. Stolz, and F. Gebhard « Exact Solution for Hopping Dissociation of Geminate Electron-Hole Pairs in a Disordered Chain », Physical review letters 100, $196602(2008)$

[14] R.Li Y. PengC.MaR.Wang, Y.WangH.Xie, T.Yang, J.Xie ,S.Yan, J.Zhang «Effect of mixture ratio on the performance of MDMO-PPV:PCBM bulk heterojunctionsolar cells: A numerical study» Materials sciebnce and engineering B Journal B172 305-310 (2010)

[15] H. Hoppe, N. S. Sariciftci and D. Meissner «Optical Constants Of Conjugated Polymer/ Fullerene Based Bulk-Heterojunction Organic Solar Cells», (2002) Mol. Cryst. Liq. Cryst., Vol. 385, pp. [233]/113-[239]/119

[16] Koeppe, R., Bossart, O., Calzaferri, G., \&Sariciftci, N. S. (2007). Advanced photon-harvesting concepts for low-energy gap organic solar cells. Sol. Energy Mater. Sol. Cells, 91(11), 986995.doi:10.1016/j.solmat.2007.01.008

[17] L. J. A. Koster, E. C. P. Smits, V. D. Mihailetchi, P. W. M. Blom, Device model for the operation of polymer/fullerene bulk heterojunction solar cells, Physical Review B, 72 (2005), 085205 